\title{
Regulation of Pigment Epithelium-Derived Factor (PEDF), an Insulin Resistance-Inducing Adipocytokine, in 3T3-L1 Adipocytes
}

Daisuke Ito, Kouichi Inukai*, Takashi Sumita, Hiraku Ono, Shigehiro Katayama and Takuya Awata

Division of Endocrinology and Diabetes, Department of Medicine, Saitama Medical School, Morohongo 38, Moroyama, Iruma-gun, Saitama, 350-0495, Japan

\begin{abstract}
Pigment epithelium-derived factor (PEDF), secreted by adipocytes, was recently demonstrated to reduce insulin sensitivity and to possibly cause insulin resistance in obesity. In the present study, we investigated the regulation of adipocyte-expressing PEDF to clarify the underlying link between obesity and PEDF expression. We examined PEDF expression in 3T3-L1 adipocytes at both the transcriptional and the protein level using RT-PCR and western blot. First, we examined PEDF expression during 3T3-L1 adipocyte differentiation. PEDF expression peaked in 3T3-L1 fibroblasts and was lowest at 2 days after initiating the induction of differentiation into adipocytes (day 2). Then, PEDF expressions gradually rose until day 10, in parallel with adipocyte differentiation. Exposure of 3T3-L1 adipocytes to a PPARa or PPARY activator reduced PEDF expression, suggesting these suppressions of PEDF expression to be involved in the mechanism by which PPAR agonists reduce insulin resistance. When 3T3-L1 adipocytes were treated with 0.5 $\mu \mathrm{M} \mathrm{H}_{2} \mathrm{O}_{2}$, PEDF levels were markedly reduced, indicating PEDF expression to be inhibited by oxidative stress. We demonstrated PEDF expression to increase in parallel with adipocyte differentiation and to be negatively regulated by oxidative stress and PPAR agonists.
\end{abstract}

Keywords: PEDF; 3T3-L1 adipocytes; Oxidative stress; Insulin resistance

Abbreviations: PEDF: Pigment Epithelium-Derived Factor; RTPCR: Real Time-Polymerase Chain Reaction; PPAR: Pperoxisome Proliferator-Activated Receptor; FHC: Ferritin Heavy Chain; ROS: Reactive Oxygen Species

\section{Introduction}

Pigment epithelium-derived factor (PEDF) is a glycoprotein belonging to the serine proteinase inhibitor superfamily [1], which has multiple functions. PEDF is known to display various effects depending on the target cell type. For example, PEDF induces apoptosis and prevents migration in endothelial cells [2], while PEDF shows protective properties [3] and differentiating activity [4] in neurons. PEDF was first identified in the eye, in cultured retinal pigment epithelial cells, and found to possess anti-angiogenic activities [4]. Decreased serum PEDF levels were found to be associated with proliferative diabetic retinopathy [5]. In addition, recent studies have shown PEDF to also be expressed at high levels in normal kidneys, and that renal PEDF is decreased in diabetic rodents [6]. Thus, PEDF is a key regulator of the mechanisms protecting against diabetic complications. PEDF is widely distributed in a variety of organs, including the eye, brain, spinal cord, liver, heart, placenta, bone, pancreas and prostate [7].

Interestingly, the serum PEDF level was recently found to be upregulated in individuals with metabolic syndrome [8] and type 2 diabetes mellitus $[9,10]$. Moreover, PEDF, as an adipocyte secretory factor, was demonstrated to reduce insulin sensitivity and is a candidate for the possible causes of insulin resistance in obesity [11]. Thus, PEDF was suggested to be among the insulin resistance-inducing adipocytokines, such as TNFa, free fatty acids (FFA) and interleukins [12-14].

Herein, we investigated the regulation of PEDF expression in 3T3L1 adipocytes at both the transcriptional and the protein level using the RT-PCR system and western blot with a PEDF-specific antibody. PEDF expression was increased in parallel with adipocyte differentiation and negative regulations were observed in response to a protein kinase $\mathrm{A}$ (PKA) activator, oxidative stress and peroxisome proliferator-activated receptor (PPAR) agonists. These results are consistent with the idea that PEDF is one of the insulin resistance-inducing adipocytokines in obesity.

\section{Methods}

\section{Cell culture and chemicals}

The 3T3-L1 fibroblasts were maintained in DMEM (Sigma-Aldrich, Co., St. Louis, MO) containing 10\% donor calf serum (Dainippon Sumitomo Pharma, Co., Osaka, Japan) at $37^{\circ} \mathrm{C}$ in $10 \% \mathrm{CO}_{2}$. Two days after the fibroblasts had reached confluence (day 0), differentiation was induced by treating the cells with DMEM containing $0.5 \mathrm{mM}$ 3-isobutyl-1-methylxanthine (Sigma-Aldrich), $4 \mu \mathrm{g} / \mathrm{ml}$ dexamethasone (Sigma-Aldrich), and 10\% fetal calf serum (FCS) (Dainippon Sumitomo Pharma) for $48 \mathrm{hr}$. Cells were re-fed with DMEM supplemented with $10 \%$ FCS for the following 8 days (days $2-10$ ). When more than $90 \%$ of the cells expressed the adipocyte phenotype (days 6-10), the cells were used for the experiments. The indicated concentrations of chemicals were added $12 \mathrm{hr}$ before cell lysate preparation. TNF- $\alpha$, palmitate, forskolin, $\mathrm{H}_{2} \mathrm{O}_{2}$ (Sigma-Aldrich), $\mathrm{H} 89$ (Seikagaku Co., Tokyo, Japan), pioglitazone (Takeda Co., Tokyo, Japan), fenofibrate (Kissei Co., Tokyo, Japan) and human recombinant adiponectin (R\&D Systems, Inc., Minneapolis, MN) were purchased. Fatty acid solution was prepared by a method described previously [15].

*Corresponding author: Kouichi Inukai, Division of Endocrinology and Diabetes, Department of Medicine, Saitama Medical School, Morohongo 38 Moroyama, Iruma-gun, Saitama, 350-0495, Japan, Fax: +81-492-76-1430; E-mail: inukai@saitama-med.ac.jp

Received October 03, 2011; Accepted November 19, 2011; Published Novembe 23, 2011

Citation: Ito D, Inukai K, Sumita T, Ono H, Katayama S, et al. (2011) Regulation of Pigment Epithelium-Derived Factor (PEDF), an Insulin Resistance-Inducing Adipocytokine, in 3T3-L1 Adipocytes. J Diabetes Metab 2:151. doi:10.4172/21556156.1000151

Copyright: @ 2011 Ito D, et al. This is an open-access article distributed unde the terms of the Creative Commons Attribution License, which permits unrestricted use, distribution, and reproduction in any medium, provided the original author and source are credited. 
Citation: Ito D, Inukai K, Sumita T, Ono H, Katayama S, et al. (2011) Regulation of Pigment Epithelium-Derived Factor (PEDF), an Insulin ResistanceInducing Adipocytokine, in 3T3-L1 Adipocytes. J Diabetes Metab 2:151. doi:10.4172/2155-6156.1000151

\section{Antibodies and western blotting}

Western blotting was performed as previously described [16]. Briefly, after incubation with the indicated chemicals, cell lysates from 3T3-L1 adipocytes were washed with ice-cold phosphate buffered saline (PBS), lysed in ice-cold lysis buffer (1\% Triton/PBS), and then centrifuged at $14,000 \mathrm{x}$ g for $10 \mathrm{~min}$ at $4^{\circ} \mathrm{C}$. After the resulting supernatants, including tissue protein extracts, had been incubated with anti-PEDF polyclonal antibody (Santa Cruz, Santa Cruz, CA), protein A-Sepharose beads (Amersham Pharmacia Biotech, Piscataway, NJ) were used to precipitate the immune complexes. The beads were washed three times with lysis buffer and the immunoprecipitated proteins were separated from the beads by boiling in Laemmli buffer. The beads were removed by centrifugation, and supernatants were subjected to SDS-polyacrylamide gel, followed by electrophoretic transfer to a nitrocellulose membrane. Membranes were incubated for $1 \mathrm{hr}$ at RT with anti-PEDF monoclonal antibody (R\&D Systems). Commercial antibodies against PU.1 and ferritin heavy chain (Santa Cruz) were also used as primary antibodies. After blotting with the indicated secondary antibody, detection was performed using an ECL chemiluminescent kit (Amersham Pharmacia Biotech) according to the manufacturer's instructions.

\section{RNA preparation and real time PCR}

Total RNA from 3T3-L1 adipocytes was isolated with Isogen (Nippon Gene, Tokyo, Japan). cDNA was synthesized from the purified RNA using a reverse transcriptase kit (Amersham Pharmacia Biotech) according to the manufacturer's instructions. For quantitative analysis of PEDF, p47 and p22 NADPH oxidase mRNA, we conducted real-time PCR using an ABI PRISM Model 7000 (Applied Biosystems, Foster City, CA) according to the manufacturer's instructions. The primer sets and probes for murine PEDF (Assay ID; Mm441270_m1), p47 and p22 NADPH oxidase (Assay ID; Mm447921_m1, Mm514478_ $\mathrm{m} 1$, respectively) were purchased.

\section{Generation and transfection of recombinant adenoviruses expressing PU.1-VP16 and ferritin heavy chain}

A full length mouse PU.1 cDNA was isolated from mouse embryo RNA by reverse transcriptase PCR. The oligonucleotide sequences used for PCR were as follows: coding sense strand sequence; 5'-GATGTTACAGGCGTGCAAAATGG-3', antisense strand sequence; 5'-ATCAGTGGGGCGGGAGGCGCCGCT-3'. The cDNAs were subcloned into TA vectors, pCRII (Invitrogen, Paisley, UK), sequenced to verify their identities and confirmed to have no unexpected mutations. VP-16 cDNA was kindly provided by H. Katagiri (Tohoku University Graduate School of Medicine) and ligated to the C-terminal portion of PU-1 cDNA with the Cla I site. Recombinant adenovirus containing full-length murine PU.1-VP16 or ferritin heavy chain (FHC) was prepared by homologous recombination of expression cosmid cassettes containing the corresponding $\mathrm{CDNAs}$ and the parental adenovirus genome, as described previously $[17,18]$. Lac $\mathrm{Z}$ adenovirus was used as a control. For adenovirus mediated transfection, 3T3-L1 adipocytes were incubated with DMEM containing the adenoviruses for $2 \mathrm{~h}$ at $37^{\circ} \mathrm{C}$ and the growth medium was then added. Experiments were performed 3 days after transfection.

\section{Detection of intracellular ROS production}

Intracellular ROS production was monitored by flow cytometry (Becton Dickinson, Franklin Lakes, NJ, USA) using 5-(and 6)-chloromethyl-2',7'-dichlorodihydrofluorescein diacetate, acetyl ester (CM- $\mathrm{H}_{2} \mathrm{DCFDA}$ ) (Invitrogen). Cells were stimulated with the indicated reagents in culture dishes and incubated for $12 \mathrm{hr}$. Then, these cells were washed twice in PBS, followed by addition of $10 \mu \mathrm{M}$ CM-H $\mathrm{H}_{2} \mathrm{DCFDA}$ in PBS, and finally placed in the dark at $37^{\circ} \mathrm{C}$ for $1 \mathrm{hr}$. CM-H ${ }_{2}$ DCFDA enters cells, is then oxidized by ROS, and stays within the cells. The cells were washed once, harvested and suspended in 500 $\mu \mathrm{l}$ of PBS. Dead cells were excluded by adding $10 \mu \mathrm{M}$ propidium iodide, a nuclear stain to which viable cells are impermeable. ROS levels were measured flow cytometrically by determining the mean fluorescent intensity relative to that of the control group. Using this method, we were able to measure not only $\mathrm{H}_{2} \mathrm{O}_{2}$, but also hydroxy radical $(\cdot \mathrm{OH})$ and peroxynitrite $\left(\mathrm{ONOO}^{-}\right)$levels.

\section{Results}

We investigated transcriptional and translational levels of PEDF expression during adipocyte differentiation of 3T3-L1 cells. First, we attempted to collect PEDF in the cell culture medium by immunoprecipitation. However, PEDF was undetectable in the immunoprecipitates by western blot. Since PEDF expression in the culture medium was apparently extremely low, we did not attempt further examination of the PEDF secreted by adipocytes. (Figure 1A) presents the changes in PEDF mRNA (1A, lower panel) and proteins (1A, upper panel) in 3T3-L1 cells from days 0 to 10 every 2 days. Interestingly, the expression levels of PEDF mRNA and protein were highest in 3T3-L1 fibroblasts (day 0 ) and lowest 2 days after changing to induction medium. Then, the expression levels of PEDF mRNA and proteins gradually increased through day 10, in parallel with adipocyte differentiation. With our experimental manipulations, over than $90 \%$ of cells expressed the adipocyte phenotype at day 6 , and both

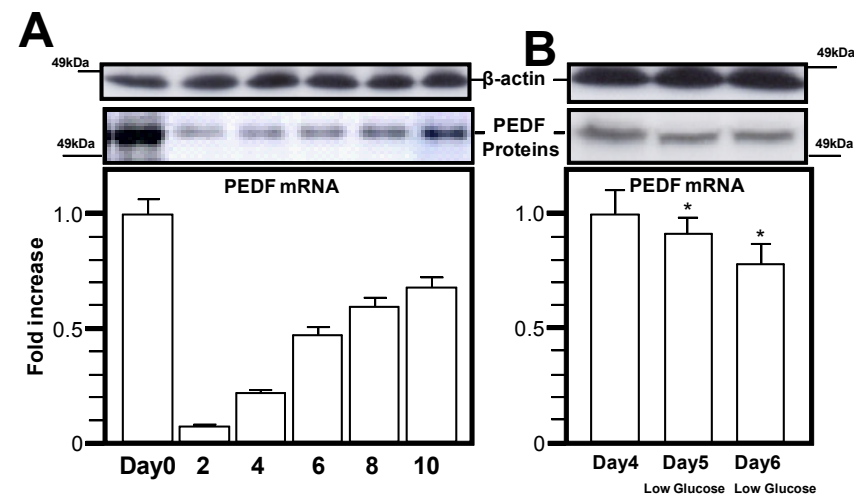

Figure 1: PEDF expression (western blot; upper panel, Q-PCR; lower panel) in 3T3-L1 adipocytes during differentiation from fibroblasts into mature adipocytes (A) and the effects of energy deprivation (B). Differentiation was induced two days after the 3T3-L1 fibroblasts had reached confluence by treating them with the indicated medium for $48 \mathrm{~h}$ (day 0 ). Cells were re-fed with FCS-containing DMEM for the following 8 days (days 2-10). To study the effects of energy deprivation, FCS-containing medium was changed to DMEM with low glucose $(1000 \mathrm{mg} / \mathrm{L})$ on day 4 . At the indicated time, cell lysates were lysed and centrifuged at $14,000 \times \mathrm{g}$ for $10 \mathrm{~min}$ at $4^{\circ} \mathrm{C}$. After the resulting supernatants, including tissue protein extracts, had been incubated with antiPEDF polyclonal antibody, protein A-Sepharose beads were used to precipitate immune complexes. After washing the beads, the immunoprecipitated proteins were subjected to SDS-PAGE, followed by electrophoretic transfer to a nitrocellulose membrane. Membranes were incubated for $1 \mathrm{hr}$ at RT with antiPEDF monoclonal antibody. After blotting with the indicated secondary antibody, detection was performed using an ECL chemiluminescent kit (upper panel). Total RNA from 3T3-L1 adipocytes was isolated with Isogen and CDNA was synthesized from purified RNA using a reverse transcriptase kit. For Q-PCR of PEDF (lower panel), we conducted real-time PCR using an ABI PRISM Model 7000. Representative data from four independent experiments are presented. *Significant difference $(P<0.05)$ relative to PEDF expression in control cells. 
Citation: Ito D, Inukai K, Sumita T, Ono H, Katayama S, et al. (2011) Regulation of Pigment Epithelium-Derived Factor (PEDF), an Insulin ResistanceInducing Adipocytokine, in 3T3-L1 Adipocytes. J Diabetes Metab 2:151. doi:10.4172/2155-6156.1000151

droplet size and cell volume of the adipocytes continued to increase after day 6. To examine whether PEDF expression is associated with energy deprivation, we changed from high glucose $(4500 \mathrm{mg} / \mathrm{L})$ to low glucose $(1000 \mathrm{mg} / \mathrm{L})$ medium at 4 days. As shown in (Figure 1B), PEDF expression decreased as incubation time increased, indicating that energy deprivation is likely to decrease PEDF expression.

Based on previous reports that PEDF is up-regulated in the serum of obese subjects [8-10], we examined whether oxidative stress, i.e. $\mathrm{H}_{2} \mathrm{O}_{2}$, palmitate (FFA) and TNFa, which are elevated in obese subjects, have effects on PEDF expression. Unexpectedly, PEDF expression was decreased by exposure to oxidative stress, $\mathrm{FFA}$ and $\mathrm{TNF}$, in a concentration-dependent manner (Figure 2A; upper panels; PEDF proteins, lower panels; PEDF mRNA). These results indicate that the serum PEDF level is increased despite PEDF expression being suppressed, via up-regulation of oxidative stress, FFA and adipocytokines, in the obese state. Next, we examined the effects of enhancing PPAR $\alpha$ and $\gamma$ activities, which have beneficial effects on insulin resistance [19,20], on PEDF expression. As shown in (Figure 2B), the PPAR $\gamma$ agonist (pioglitazone) and the PPAR a agonist (fenofibrate) inhibited PEDF expression by approximately $40 \%$ and $30 \%$, respectively. These results indicate that the suppression of PEDF expression by PPAR agonists is involved in the mechanism by which PPAR agonists reduce insulin resistance.

We further examined the effects of adiponectin, a PKA activator and components of the induction medium, i.e., insulin and dexamethasone, on PEDF expression. When incubated with $20 \mu \mathrm{g} / \mathrm{ml}$ full-length adiponectin and $200 \mu \mathrm{M}$ forskolin, a PKA activator, marked reductions of both mRNA and proteins of PEDF were observed (Figure 2C and D). This effect of forskolin was partially abolished by the addition of H89, a PKA inhibitor, suggesting that PEDF expression was inhibited by enhancing PKA activity. We previously presented data showing that a PKA activator stimulated PPAR $\gamma$ activity in 3T3-L1 adipocytes [21], which may explain the mechanism by which PEDF expression is inhibited by a PKA activator. The induction medium components, i.e., insulin or dexamethasone, did not alter PEDF expression significantly, indicating that PEDF expression is not affected by insulin signaling (Figure 2E).

To confirm the effects of oxidative stress on PEDF expression more precisely, we adopted a method which regulates ROS directly by inducing recombinant proteins via adenovirus transfection. We first prepared the adenovirus expressing PU.1-VP16, which is a constitutively active form of the PU.1 transcription factor. With overexpression of PU.1VP16 in 3T3-L1 adipocytes, p22 or p47 NADPH oxidase subunits were markedly increased depending on the expression level of PU.1-VP16 proteins (Figure 3A). Then, we evaluated ROS accumulation in the presence or absence of FFA by employing the ROS assay. As shown in the upper panel of (Figure 3B), ROS accumulation was significantly increased in the presence of FFA, while no increase was observed in the absence of FFA. These results suggest that the constitutively active PU.1 transcription factor promotes ROS production only in the presence of substrates, i.e., FFA. Under these conditions, we analyzed PEDF expression. As expected, the PEDF transcriptional level was decreased in parallel with ROS accumulation (Figure 3B, lower panel). We also prepared adenovirus expressing recombinant FHC proteins, which reduce ROS accumulation as previously described [18]. With overexpression of FHC proteins, ROS accumulation, which was elevated in the presence of $0.5 \mu \mathrm{M} \mathrm{H}_{2} \mathrm{O}_{2}$, was completely abolished (Figure 4A). Under these conditions, we similarly analyzed the transcriptional levels of PEDF (Figure 4B). The reducing effects of $\mathrm{H}_{2} \mathrm{O}_{2}$ on PEDF expression were partially or completely restored when FHC proteins were overexpressed, suggesting the level of oxidative stress to directly reduce PEDF expression.

\section{Discussion}

A number of studies described PEDF as having various functional roles [2-6]. Among these, PEDF plays crucial roles in preventing diabetic complications, because its anti-angiogenic activity is exerted via inhibition of retinal angioneogenesis [5] and its anti-fibrogenic activity prevents the progression of diabetic nephropathy [6,22]. Thus, PEDF was thought for several years to be one of the major proteins in type 2 diabetes. However, this idea was recently challenged by two reports indicating circulating levels of PEDF to be up-regulated in patients with type 2 diabetes [9] and also that PEDF is involved in obesity-induced insulin resistance [11]. Though the precise mechanism by which PEDF triggers insulin resistance is unclear, the insulin mediated IRS-1 phosphorylation in muscle and liver is attenuated by PEDF administration [11]. In this study, we investigated the regulation of PEDF expression in 3T3-L1 adipocytes and found that oxidative stress accumulation to suppress PEDF expression. Although our results are likely to be inconsistent with the relatively high serum PEDF levels in obese people, this might be explained by the serum level of an adipocytokine not necessarily correlating with its intracellular expression level. Resistin, which is also an insulin resistance-inducing adipocytokine, is a good example. Though serum circulating resistin is elevated in obese mice, its expression per adipose tissue weight is actually reduced in this murine model [23]. While PEDF expression per adipose tissue weight is reduced by factors such as oxidative stress, FFA and TNFa, the overall amount of PEDF secreted per adipose tissue weight may actually be increased, resulting in the high serum circulating levels of PEDF, as well as resistin, in obese subjects.

A

B

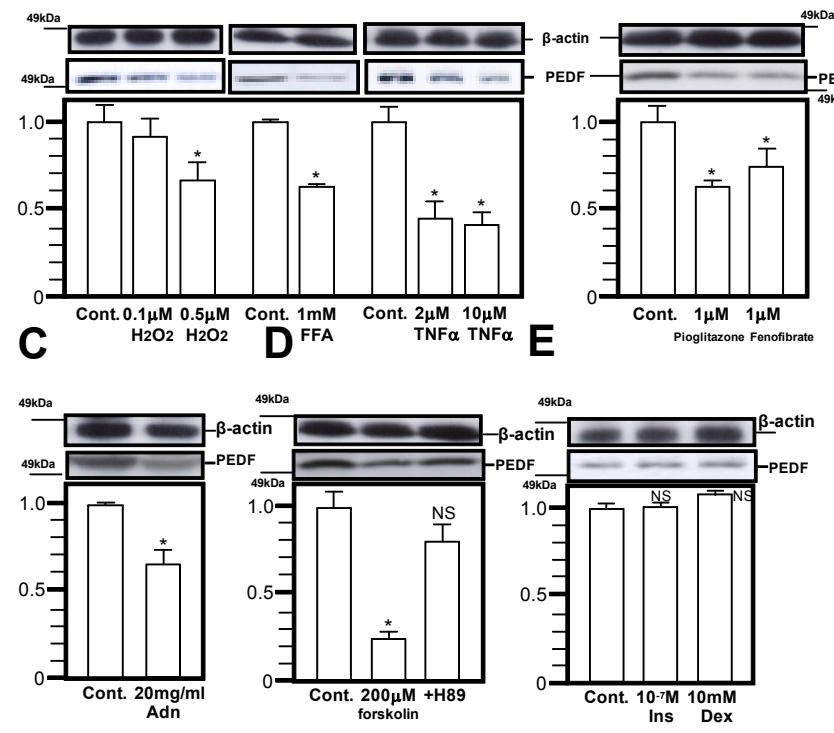

Figure 2: PEDF expression (western blot; upper panel, Q-PCR; lower panel) in 3T3-L1 adipocytes with $12 \mathrm{hr}$ incubation using $\mathrm{H}_{2} \mathrm{O}_{2}$, FFA and TNFa (A), PPAR agonists (B), adiponectin (C), PKA agonists (D), insulin and dexamethasone $(E)$. The mature 3T3-L1 adipocytes (days 6-10) were incubated with the indicated chemicals for $12 \mathrm{hr}$, followed by western blot (upper panel) and Q-PCR (lower panel). Representative data from four independent experiments are presented. *Significant difference $(P<0.05)$ relative to PEDF expression in control cells. N.S.; not significant relative to control cells. 
A
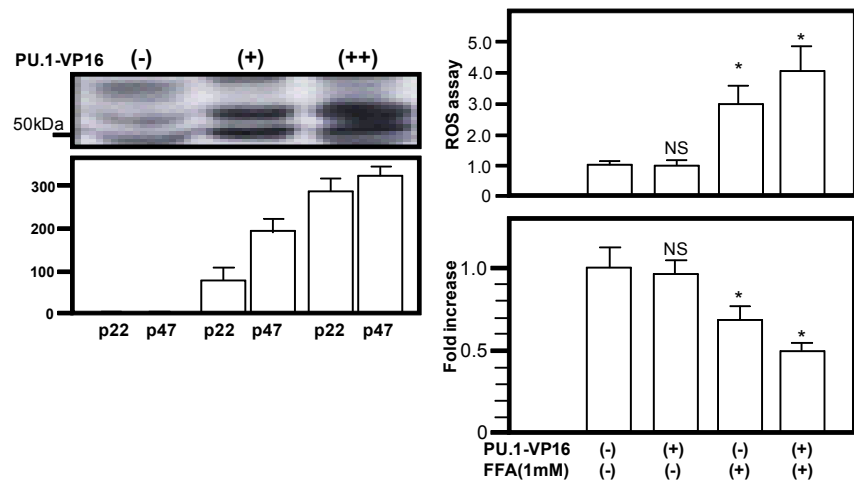

Figure 3: PEDF expression with up-regulation of NADPH oxidase. The adenovirus expressing the constitutively active form of PU.1 transcription factor was transfected into 3T3-L1 adipocytes and levels of PU.1 protein expression were measured ( $A$, upper panel), as well as transcription levels of two isoforms of NADPH oxidase, p22 and p47 (A, lower panel). After stimulating 3T3-L1 adipocytes with $1 \mathrm{mM}$ FFA for $12 \mathrm{hr}$, these cells were washed twice in PBS, followed by addition of $10 \mu \mathrm{M} \mathrm{CM}-\mathrm{H}_{2}$ DCFDA in PBS, placed in the dark at $37^{\circ} \mathrm{C}$ for $1 \mathrm{hr}$, then harvested and suspended in $500 \mu \mathrm{l}$ of PBS. ROS levels were measured flow cytometrically by determining the mean fluorescent intensity relative to that of the control group $(B$, upper panel). Corresponding to the cells used for the ROS assay, PEDF expression was examined by Q-PCR (B, lower panel). Representative data from four independent experiments are presented. *Significant difference $(P<0.05)$ relative to control cells. N.S.; not significant relative to control cells.
A

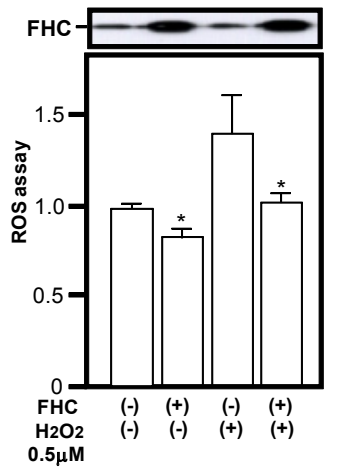

B

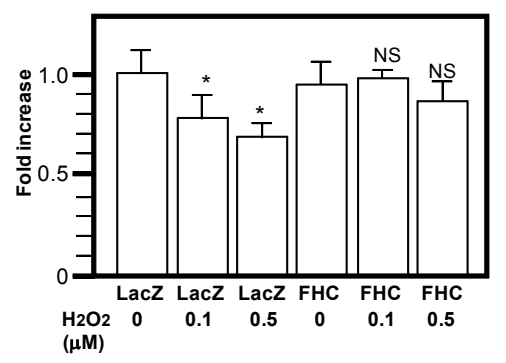

Figure 4: PEDF expression with FHC overexpression. The adenovirus expressing Lac Z or FHC was transfected into 3T3-L1 adipocytes and levels of $\mathrm{FHC}$ protein expression were confirmed (A, upper panel). ROS levels in the presence or in the absence of $0.5 \mu \mathrm{M} \mathrm{H}_{2} \mathrm{O}_{2}$ were measured flow cytometrically by determining the mean fluorescent intensity relative to that of the control group (A, lower panel). PEDF expression in the cells was examined by Q-PCR (B). Representative data from four independent experiments are presented. *Significant difference $(P<0.05)$ relative to control cells. N.S.; not significant relative to control cells.

PEDF expression was decreased by incubation in low glucose medium, indicating that energy deprivation is likely to decrease PEDF expression. A recent study by Franck et al. [24] showed mRNA and serum levels of PEDF to be decreased by caloric restriction and increased by refeeding and overfeeding. Thus, the decrease in PEDF expression in response to decreased glucose levels observed in our study may reflect PEDF regulation via energy availability being independent of fat accumulation, as demonstrated by the Franck et al study. Our results also explain one of the mechanisms by which pioglitazone relieves insulin resistance. To date, many studies have tackled this issue, and demonstrated pioglitazone to inhibit the expressions of a number of substances which induce insulin resistance, including TNFa, FFA, resistin, etc $[20,23]$. Our results support the idea that suppression of PEDF expression in adipose tissue is a mechanism through which pioglitazone contributes to improved insulin resistance. Further studies, investigating serum PEDF level changes in type 2 diabetic patients being treated with pioglitazone, are needed clarify this issue.

To confirm the effects of oxidative stress on PEDF expression, we employed adenovirus expressing oxidative stress-related genes. $\mathrm{NADPH}$ oxidase expression was reported to be augmented in adipose tissue of genetically obese mice and to be related to oxidative stress production [25]. Thus, to artificially mimic the condition of these mice by increasing NADPH oxidase expression, we overexpressed constitutively activated PU-1 transcription factor in 3T3-L1 adipocytes. The promoter activity of the $\mathrm{p} 47$ (phox) isoform of NADPH oxidase was previously reported to be enhanced by PU.1 in myeloid cells [26]. In this study, though we demonstrated marked increases only in the p22 and p47 NADPH isoforms, other isoforms were also increased (data not shown). Under these conditions, not only $\mathrm{H}_{2} \mathrm{O}_{2}$, but also other ROS (superoxide anion, etc) are increased by up-regulation of NADPH oxidase in the presence of FFA. In addition, we feel that our methods are more useful for evaluating the physiological effects of oxidative stress than the addition of $\mathrm{H}_{2} \mathrm{O}_{2}$, which is very toxic to the cells. We also transfected adipocytes with the adenovirus expressing FHC, an antioxidative protein, which was demonstrated to reduce ROS in our previous study [18]. As this antioxidative protein has a crucial role in balancing the accumulation of oxidative stress, it is important to observe the physiological effects of oxidative stress by modulating the expressions of antioxidative proteins.

In conclusion, we examined the regulation of PEDF expression in 3T3-L1 adipocytes and found this expression to be regulated by adipocyte differentiation, exposure to PPAR agonists and oxidative stress, which may partially explain the triggering or negative feedback systems against insulin resistance in adipose tissues of obese subjects.

\section{References}

1. Steele FR, Chader GL, Johnson LV, Tombran-Tink J (1992) Pigment epitheliumderived factor: neurotrophic activity and identification as a member of the serine protease inhibitor gene family. Proc Natl Acad Sci USA 90: 1526-1530.

2. Volpert OV, Zaichuk T, Zhou W, Reiher F, Ferguson TA, et al. (2002) Inducerstimulated Fas targets activated endothelium for destruction by anti-angiogenic thrombospondin-1 and pigment epithelium-derived factor. Nat Med 8: 349-357.

3. Taniwaki T, Becerra SP, Chader GL, Schwartz JP (1995) Pigment epitheliumderived factor is a survival factor for cerebellar granule cells in culture. $J$ Neurochem 64: 2509-2517.

4. Tombran-Tink J, Chader CG, Johnson LV (1991) PEDF: pigment epitheliumderived factor with potent neuronal differentiative activity. Exp Eye Res 53: 411-414.

5. Spranger J, Osterhoff M, Reimann M, Mohlig M, Ristow M, et al. (2001) Loss of anti-angiogenic pigment epithelium-derived factor in patients with angiogenic eye disease. Diabetes 50: 2641-2645

6. Wang JJ, Zhang SX, Lu K, Chen Y, Mott R, et al. (2005) Decreased expression of pigment epithelium-derived factor is involved in the pathogenesis of diabetic nephropathy. Diabetes 54: 243-250.

7. Tombran-Tink J, Mazuruk K, Rodriguez IR, Chung D, Linker T, et al. (1996) Organization, evolutionary conservation, expression and unusual Alu density of the human gene for pigment epithelium-derived factor, a unique neurotrophic serpin. Mol Vis 2: 11

8. Yamagishi S, Adachi H, Abe A, Yashiro T, Enomoto M, et al. (2006) Elevated 
Citation: Ito D, Inukai K, Sumita T, Ono H, Katayama S, et al. (2011) Regulation of Pigment Epithelium-Derived Factor (PEDF), an Insulin ResistanceInducing Adipocytokine, in 3T3-L1 Adipocytes. J Diabetes Metab 2:151. doi:10.4172/2155-6156.1000151

Page 5 of 5

serum levels of pigment epithelium-derived factor in the metabolic syndrome. $\mathrm{J}$ Clin Endocrinol Metab 91: 2447-2450.

9. Jenkins A, Zhang SX, Gosmanova A, Aston C, Dashti A, et al. (2008) Increased serum Pigment Epithelium Derived Factor levels in Type 2 diabetes patients. Diabetes Res Clin Pract 82: e5-e7.

10. Ogata N, Matsuoka M, Matsuyama K, Shima C, Tajika A, et al. (2007) Plasma concentration of pigment epithelium-derived factor in patients with diabetic retinopathy. J Clin Endocrinol Metab 92: 1176-1179.

11. Crowe S, Wu LE, Economou C, Turpin SM, Matzaris M, et al. (2009) Pigment epithelium-derived factor contributes to insulin resistance in obesity. Cell Metab 10: 40-47.

12. Hotamisligil GS, Shargill NS, Spiegelman BM (1993) Adipose expression of tumor necrosis factor-alpha: direct role in obesity-linded insulin resistance. Science 259: 87-91.

13. Jongh RT, Serné EH, Ljzerman RG, Vries G, Stehouwer CD (2004) Free fatty acid levels modulate microvascular function: relevance for obesity-associated insulin resistance, hypertension and microangiopathy. Diabetes 53: 2873-2882.

14. Kern PA, Ranganathan S, Li C, Wood L, Ranganathan G (2001) Adipose tissue tumor necrosis factor and interleukin- 6 expression in human obesity and insulin resistance. Am J Physiol Endocrinol Metab 280: E745-E751.

15. Sinha S, Perdomo G, Brown NF, O'Doherty RM (2004) Fatty acid-induced insulin resistance in L6 myotubes is prevented by inhibition of activation and nuclear localization of nuclear factor kappa B. J Biol Chem 279: 41294-41301.

16. Inukai K, Shewan AM, Pascow WS, Katayama S, James DE, et al. (2004) Carboxy terminus of glucose transporter 3 contains an apical membrane targeting domain. Mol Endocrinol 18: 339-349.

17. Miyake S, Makimura M, Kanegae Y, Harada S, Sato Y, et al. (1996) Efficient generation of recombinant adenoviruses using adenovirus DNA-terminal protein complex and a cosmid bearing the full-length virus genome. Proc Nat Acad Sci USA 93: 1320-1324.

18. Ikegami Y, Inukai K, Imai K, Sakamoto Y, Katagiri H, et al. (2009) Adiponectin upregulates ferritin heavy chain in skeletal muscle cells. Diabetes 58: 61-70.

19. Guerre-Millo M, Gervois P, Raspe E, Madsen L, Poulain P, et al. (2000) Peroxisome proliferators-activated receptor alpha activators improve insulin sensitivity and reduce adiposity. J Biol Chem 275: 16638-16642.

20. Miyazaki Y, Mahankali A, Wajcberg E, Bajaj M, Mandarino LJ, et al. (2004) Effects of pioglitazone on circulating adipocytokine level and insulin sensitivity in type 2 diabetic patients. J Clin Endocrinol Metab 89: 4312-4319.

21. Watanabe M, Inukai K, Katagiri H, Awata T, Oka Y, et al. (2002) Regulation of PPARy transcriptional activity in 3T3-L1 adipocytes. Biochem Biophys Res Commun 300: 429-436.

22. Wang JJ, Zhang SX, Motto R, Knapp RR, Cao W, et al. (2006) Salutary effect of pigment epithelium-derived factor in diabetic nephropathy. Diabetes 55: 16781685.

23. Rajala MW, Yong Q, Patel HR, Takahashi N, Banerjee R, et al. (2004) Regulation of resistin expression and circulating levels in obesity, diabetes and fasting. Diabetes 53: 1671-1679.

24. Franck N, Gummesson A, Jernås M, Glad C, Svensson PA, et al. (2011) Identification of adipocyte genes regulated by caloric intake. J Clin Endocrino Metab 96: E413-E418.

25. Furukawa S, Fujita T, Shimabukuro M, Iwaki M, Yamada Y, et al. (2004) Increased oxidative stress in obesity and its impact on metabolic syndrome. $J$ Clin Invest 114: 1752-1761.

26. Li SL, Valente AJ, Zhao SJ, Clark RA (1997) PU.1 is essential for p47(phox) promoter activity in myeloid cells. J Biol Chem 272: 17802-17809. 